\title{
Astrometry of Single-Chord Occultations: Application to the 1993 Triton Event
}

\author{
Catherine B. Olkin, ${ }^{1}$ J. L. Elliot, ${ }^{1,2}$ Schelte J. Bus, and Stephen W. MCDonald \\ Department of Earth, Atmospheric and Planetary Sciences, Massachusetts Institute of Technology, Cambridge, \\ Massachusetts $02139-4307$ \\ Electronic mail: (colkin, jim, sjb, mcdonald)@astron.mit.edu \\ CONARD C. DAHN \\ US Naval Observatory, P.O. Box 1149, Flagstaff, Arizona 86002-1149 \\ Electronic mail: dahn@nofs.navy.mil \\ Received 1995 August 18; accepted 1995 November 27
}

\begin{abstract}
This paper outlines a method for reducing astrometric data to derive the closest approach time and distance to the center of an occultation shadow for a single observer. The method applies to CCD frames, strip scans or photographic plates and uses a set of field stars of unknown positions to define a common coordinate system for all frames. The motion of the occulting body is used to establish the transformation between this common coordinate system and the celestial coordinate system of the body's ephemeris. This method is demonstrated by application to the Tr60 occultation by Triton on 1993 July 10 UT. Over an interval of four nights that included the occultation time, 80 frames of Triton and Tr60 were taken near the meridian with the U.S. Naval Observatory (USNO) 61-inch astrometric reflector. Application of the method presented here to these data yields a closest approach distance of $359 \pm 133 \mathrm{~km}$ (corresponding to $0.017 \pm 0.006$ arcsec) for the occultation chord obtained with the Kuiper Airborne Observatory (KAO). Comparison of the astrometric closest approach time with the KAO light-curve midtime shows a difference of $2.2 \pm 4.1 \mathrm{~s}$. Relative photometry of Triton and $\operatorname{Tr} 60$, needed for photometric calibration of the occultation light curve, is also presented.
\end{abstract}

\section{INTRODUCTION}

From an occultation observation, one can learn about planetary rings and atmospheres with kilometer-scale spatial resolution (Elliot 1979), but to take full advantage of these data requires accurate knowledge of the location of the observed chord relative to the center of the occultation shadow. This must be known with an accuracy (ideally better than a few kilometers) such that the uncertainty introduced by astrometric errors into the physical parameters derived from the light curve is smaller than their formal errors from model fitting. The most reliable method for determining the location of an occultation chord relative to the center of the shadow has been to use the immersion and emersion times from a set of occultation chords to fit a two-dimensional figure to the body shadow. For the giant planet atmospheres, which are rapidly rotating, an oblate figure is fit to the "half-light" times (French et al. 1985; Baron et al. 1989). For a slowly rotating atmosphere, such as Pluto, a spherical model is adequate (Millis et al. 1993). For large, airless bodies, such as Pallas, an elliptical figure has been used (Wasserman et al. 1979), but with a larger number of chords, more details can be inferred about the figure of the body (Dunham et al. 1990).

Other methods for determining the location of the occultation chord relative to the center of the occultation shadow

\footnotetext{
'Also at Lowell Observatory, Flagstaff, AZ 86001-4499.

${ }^{2}$ Also at Department of Physics, Massachusetts Institute of Technology, Cambridge, MA 02139-4307.
}

include (for Saturn and Uranus) using ring occultations to establish the astrometry and learn where in the atmosphere the chord probed (Baron et al. 1989; Nicholson et al. 1995), since the orbits of the rings are known quite accurately (French et al. 1988; Elliot et al. 1993). Also, a central flash can be used to establish the position of a single chord (Lellouch et al. 1986). Finally, one can use the length of the chord along with the known figure of the body at a specific pressure level (Sicardy et al. 1991) to achieve the same end. However, in using that method, one must assume that the atmospheric structure is the same at the time for which the figure was established and at the time of the single-chord observation.

For the Triton occultation presented here, we have only one observed chord (from the KAO), so the standard method of fitting immersion and emersion times from multiple chords cannot be used for the astrometric calibration. We were fortunate to obtain high-quality astrometric data to predict the occultation. These data produced such an accurate prediction that we were led to investigate just how well they could be used in a post-event analysis, especially since additional data were recorded the night after the occultation as well.

We refer to the method presented here as the ephemeris method for reasons that will become apparent. We make the basic assumption that stars do not move significantly over a time span of a few days and use a common set of field stars to define a reference system (in detector coordinates) across all frames. We can use the occulting body's ephemeris to set 
the scale in right ascension and declination. With this approach we do not have to reconcile two or more systems of celestial coordinates (the occulting body's ephemeris and that of an astrometric network), and we are immune to scale errors that must be present at some level in an astrometric network.

We shall first describe the method for determining the placement of the observed chord relative to the center of the occulting body from astrometric data. The next section describes the observations and details of the astrometric and photometric calibration for the Tr60 occultation (McDonald and Elliot 1992). Finally, we present a discussion of the error and considerations for application of this method to future occultations.

\section{OVERVIEW OF THE EPHEMERIS METHOD}

The method outlined here can be applied to CCD stare frames, CCD strip scans or photographic plates. Some of the frames (or plates) should contain the occulting body and occulted star both before and after the occultation event. Additional frames, without the occulting body, can be used to reduce the error in the occulted star center, but this is not typically the leading source of error. Also, for best results (highest precision) the mean center of the occulting body observations should be as close to the occulted star center as possible.

This paper deals solely with the reduction of stare frames. This analysis could be applied to strip scans, but the low frequency errors in the star positions (Dunham et al. 1991) would have to be removed first. Strip scan data used for the occultation prediction were not used in this analysis because of a field distortion found in the data (Dunham, personal communication).

The ephemeris method is based on three assumptions. The first is that the field stars used for the common reference system are fixed (over the time scale of the observations-a few days). The second assumption is that the occulting body's motion can be used to set the scale in right ascension and declination. The ephemeris does not have to be correct in terms of absolute coordinates because we are only interested in relative positions. The final assumption is that displacements of observed positions of the stars and occulting body relative to the mean centers (the centers of the stars without displacing effects such as refraction and aberration) can be removed by a linear registration. We will break up the sources of these displacements into two categories: (i) known effects such as refraction, diurnal aberration, annual aberration, and general relativistic bending, and (ii) unknown nonlinear effects such as field distortion of the telescope, detector distortion, nonuniform thermal distortion or significant chromatic differential refraction.

In the following discussion, we use the term registration of a set of positions to a coordinate system to mean a leastsquares fit that determines the linear transformation coefficients [which we shall also call registration coefficients; see Eq. (2) below].

This analysis is based on the construction of a common reference system-the detector network, which is the weighted average of the centers of each field star chosen for the reference system. Even if some frames are missing reference stars (due to poor seeing, or inconsistent centering causing stars at the edges to be lost), the mean frame can still be constructed. Each frame is linearly registered to the detector network to transform the occulted star and occulting body centers to a common reference system. The origin of this system is shifted to be the weighted mean center of the occulted star. Next, the scale and center of the detector network are related to celestial coordinates through a linear registration of the occulting body centers to the ephemeris. This allows us to determine the right ascension and declination of the occulted star in the coordinate system defined by the body's ephemeris. From here, we can determine the closest approach of the occulting body to the star and therefore, the placement of the occultation chord to the center of the body's shadow.

\section{DESCRIPTION OF THE METHOD}

In this section we present the method in detail. First, one establishes a set of stars that are common to all $N_{f}$ frames and determines their centers by a suitable procedure. To be definite, we shall refer to CCD data and the row and column center of the $n$th star in the $k$ th frame as $\left(r_{n k}, c_{n k}\right)$. For photographic plates, one might choose a different notation. For the $k$ th frame, the row and column coordinates of the occulted star are denoted by $\left(r_{* k}, c_{* k}\right)$, and those for the occulting body (in many cases, a "planet") by $\left(r_{p k}, c_{p k}\right)$.

\subsection{Creating the Detector Network}

A common coordinate system for all the frames is needed, so a detector network is created from the weighted average position for each of the selected field stars. We weight the data by frame, since the frames are not necessarily the same quality due to varying observing conditions (seeing, airmass). For the data used here, the errors in the star positions appear to be the same for a single frame, independent of stellar magnitude (over the magnitude range of 12.6 to 18.7 from a yellow photographic emulsion, kindly supplied by $\mathrm{A}$. Klemola). For this case, the weights, $w_{r k}$ for the rows and $w_{c k}$ for the columns, are derived from a registration of each frame to an unweighted detector network. For the $n$th star we find its coordinates in the unweighted detector network by the weighted averages (with all the weights set equal to 1):

$$
\begin{gathered}
\bar{r}_{n}=\frac{\sum_{k=1}^{N_{f}} w_{r k} r_{n k}}{\sum_{k=1}^{N_{f}} w_{r k}}, \\
\vec{c}_{n}=\frac{\sum_{k=1}^{N_{f}} w_{c k} c_{n k}}{\sum_{k=1}^{N_{f}} w_{c k}} .
\end{gathered}
$$

Then each frame is registered to the unweighted detector network, so that the reciprocal of the variance of the row and column residuals from the least-squares fit can be used as the row and column weights for each frame, and Eq. (1) is used a second time. The result is the detector network.

For each frame, the row and column positions of the network stars are registered to the detector network to ci +ormine $^{+}$ the registration coefficients, $a$. 


$$
\begin{gathered}
r_{m k}=a_{1 k}+a_{2 k} r_{n k}+a_{3 k} c_{n k} \\
c_{m k}=a_{4 k} a_{5 k} r_{n k}+a_{6 k} c_{n k}
\end{gathered}
$$

The observed row and column centers for the occulting body and occulted star are transformed to the detectornetwork coordinate system with the registration coefficients found in $\mathrm{Eq}$. (2). The resulting occulting body coordinates for the $k$ th frame are denoted by $\left(r_{r p k}, c_{r p k}\right)$. Similarly, the occulted star coordinates in this detector-network system are $\left(r_{r} *_{k}, c_{r * k}\right)$.

\subsection{Relating the Mean Frame to Celestial Coordinates}

Since the row-column coordinates of the detector are in a plane, we convert the celestial coordinates of right ascension and declination (RA, $\alpha$, and Dec. $\delta$ ) to those in a plane tangent to the celestial sphere (assumed parallel to the detector). The optical axis of the telescope intersects the meanframe coordinate system at the center of the detector which correspond to celestial coordinates $\left(\alpha_{0}, \delta_{0}\right)$. Here we introduce the tangent plane, as defined by Smart (1977), that has the coordinate $\xi$ parallel to RA and $\eta$ parallel to Dec. The conversion equations between $(\alpha, \delta)$ and $(\xi, \eta)$ are (Smart 1977):

$$
\begin{aligned}
& \xi=\frac{\cos \delta \sin \left(\alpha-\alpha_{0}\right)}{\sin \delta_{0} \sin \delta+\cos \delta_{0} \cos \delta \cos \left(\alpha-\alpha_{0}\right)} \\
& \eta=\frac{\cos \delta_{0} \sin \delta-\sin \delta_{0} \cos \delta \cos \left(\alpha-\alpha_{0}\right)}{\sin \delta_{0} \sin \delta+\cos \delta_{0} \cos \delta \cos \left(\alpha-\alpha_{0}\right)}
\end{aligned}
$$

As previously mentioned, we use the body's ephemeris to establish the $(\alpha, \delta)$ system. The ephemeris should be topocentric and include a correction for light-travel time, but may or may not contain corrections for diurnal and annual aberration (see Sec. 4). Due to our third assumption (that the effects of refraction, diurnal aberration, annual aberration, and other displacements of the star centers can be removed by a linear registration), these corrections can either be included or not, since they will not make a difference in the resulting relative position of the occulting body and occulted star.

We generate $\left(\alpha_{e k}, \delta_{e k}\right)$ from the ephemeris at the midtimes $t_{k}$, of the frames where the subscript " $e$ " stands for ephemeris. From $\left(\alpha_{e k}, \delta_{e k}\right)$ we use Eq. (3) to calculate the tangent-plane coordinates $\left(\xi_{e k}, \eta_{e k}\right)$ for the occulting body as given by the ephemeris. The registration of these tangentplane coordinates to the occulting body's positions in the detector-network coordinate system provide the transformation to celestial coordinates (as defined by the ephemeris of the body).

We center this transformation on the occulted star. To do this we form the weighted average row and column position in the detector-network system. We denote this center by $\left(\left\langle r_{r} * k\right\rangle,\left\langle c_{r} * k\right\rangle\right)$, and it is computed with the weights $\left(w_{r k}, w_{c k}\right)$. This shift of origin does not change the solution; it only simplifies later equations [Eqs. (5) and (8)].

We perform a linear least-squares fit of the body observations in the detector-network system to the ephemeris posi- tions and solve for the registration coefficients $(b)$, see Eq. (4). This least-squares fit is performed with the appropriate weights, $\left(w_{r k}, w_{c k}\right)$.

$$
\begin{aligned}
& \xi_{e k}=b_{1}+b_{2}\left(r_{r p k}-\left\langle r_{r * k}\right\rangle\right)+b_{3}\left(c_{r p k}-\left\langle c_{r * k}\right\rangle\right), \\
& \eta_{e k}=b_{4}+b_{5}\left(r_{r p k}-\left\langle r_{r * k}\right\rangle\right)+b_{6}\left(c_{r p k}-\left\langle c_{r * k}\right\rangle\right) .
\end{aligned}
$$

Given the registration parameters of Eq. (4), the tangent plane coordinates of the occulted star can be easily calculated:

$$
\begin{aligned}
& \xi_{*}=b_{1}+b_{2}\left\langle r_{r * k}-\left\langle r_{r * k}\right\rangle\right\rangle+b_{3}\left\langle c_{r * k}-\left\langle r_{r * k}\right\rangle\right\rangle=b_{1}, \\
& \eta_{*}=b_{4}+b_{5}\left\langle r_{r * k}-\left\langle r_{r * k}\right\rangle\right\rangle+b_{6}\left\langle c_{r * k}-\left\langle c_{r * k}\right\rangle\right\rangle=b_{4} .
\end{aligned}
$$

Now we can calculate the right ascension and declination of the occulted star using the transformation from tangent plane to celestial coordinates given by Smart (1977):

$$
\begin{gathered}
\tan \left(\alpha_{*}-\alpha_{0}\right)=\frac{\xi_{*}}{\cos \delta_{0}-\eta_{*} \sin \delta_{0}}, \\
\tan \delta_{*}=\frac{\sin \delta_{0}+\eta_{*} \cos \delta_{0}}{\cos \delta_{0}-\eta_{*} \sin \delta_{0}} \cos \left(\alpha_{*}-\alpha_{0}\right) .
\end{gathered}
$$

This is the end result of our astrometric reductions. From here, the shadow path can be determined by the star's right ascension and declination and the planet's ephemeris (the same one used in the registration above).

\subsection{Calculating the Impact Parameter and Time}

To calculate the impact parameter (or closest approach distance between the star and occulting body) we define a Cartesian coordinate system $(f g h)$, with its origin at the center of the Earth, with $f$ pointing in the direction of increasing right ascension and $h$ pointing to the occulted star (Elliot et al. 1993). We construct the $f g h$ coordinates of any object by creating its $X Y Z$ position from the object's right ascension. declination and distance, $d$, and then rotating the $X Y Z$ coordinates to $f g h$. The rotation matrix to convert from $X Y Z$ to $f g h$ is a function of the right ascension and declination of the occulted star (see Elliot et al. 1993). The conversion from the $(\xi, \eta)$ plane to the $(f, g)$ plane for an object at a distance $d$ is $f=d \xi$ and $g=d \eta$ when the same center, $\left(\alpha_{0}, \delta_{0}\right)$ is used as the origin for each coordinate system.

To find the geocentric impact parameter, we convert a geocentric planet ephemeris (with a light-travel time correction) to $f g h$ coordinates. In this coordinate system, we define the impact parameter, $p$, as the minimum distance between the center of the occulting body's shadow and the observer. In the $f g h$ system, $f_{0}(t)$ and $g_{0}(t)$ are the coordinates of the observer (Elliot et al. 1993), and $f_{p}(t)$ and $g_{p}(t)$ are the coordinates of the center of the occulting body's shadow as a function of time. The impact parameter is given by

$$
p=\operatorname{Min}\left\{\sqrt{\left[f_{0}(t)-f_{p}(t)\right]^{2}+\left[g_{0}(t)-g_{p}(t)\right]^{2}}\right\} .
$$

The minimization denoted by Eq. (7) gives the impact parameter, and the time of the minimum is the predicted closest approach time. The latter can be compared with the midtime of the occultation light curve as a test of the astrometric solution. 


\subsection{Errors}

Here we calculate the errors in the impact parameter and occultation midtime, according to the assumptions of our astrometric reduction procedure. We begin with the errors expressed in the tangent plane-derived from Eq. (5), in which $\xi_{*}$ and $\eta_{*}$ are each expressed as a sum of three terms. There is no correlation between the offset term $\left(b_{1}\right)$ and the slope terms $\left(b_{2}\right.$ and $\left.b_{3}\right)$ due to the choice of $\left(\left\langle r_{r} * k\right\rangle,\left\langle c_{r} * k\right\rangle\right)$ as an offset for the registration. Also the two slopes terms are assumed to be uncorrelated because they are in orthogonal directions. We denote the variance of the sample means $\left\langle r_{r} * k\right\rangle,\left\langle c_{r} * k\right\rangle$ by $\sigma^{2}\left(\left\langle r_{r} * k\right\rangle\right), \sigma^{2}\left(\left\langle c_{r} * k\right\rangle\right)$. Taking the appropriate partial derivatives, we find that the variances $\sigma^{2}\left(\xi_{*}\right), \sigma^{2}\left(\eta_{*}\right)$ are:

$$
\begin{aligned}
& \sigma^{2}\left(\xi_{*}\right)=\sigma^{2}\left(b_{1}\right)+b_{2}^{2} \sigma^{2}\left(\left\langle r_{r_{k}}\right\rangle\right)+b_{3}^{2} \sigma^{2}\left(\left\langle c_{r * k}\right\rangle\right), \\
& \sigma^{2}\left(\eta_{*}\right)=\sigma^{2}\left(b_{4}\right)+b_{5}^{2} \sigma^{2}\left(\left\langle r_{r_{k} *}\right\rangle\right)+b_{6}^{2} \sigma^{2}\left(\left\langle c_{r * k}\right\rangle\right) .
\end{aligned}
$$

We can gain some understanding of these errors by considering a case where the $\xi$ axis is perfectly aligned with the row axis so that $b_{3}=b_{5}=0$. We work only with the equation for $\xi$, since the $\eta$ result is completely analogous. Using the equations for linear-least squares (Clifford 1973), we can write an expression for the variance of $b_{1}$ in Eq. (8) in terms of the row weights, $w_{r k}$, the measurements of the occulting body's position, $\xi_{e}\left(t_{k}\right)$, and the scale factor in radians per pixel, $b_{2}$. First, we define the ephemeris position in the detector-network system:

$$
r_{r e}\left(t_{k}\right)=\frac{\xi_{e}\left(t_{k}\right)}{b_{2}}+\left\langle r_{r * k}\right\rangle
$$

The variance of the sample mean of the star and the equivalent quantity for the occulting body (this is equal to the variance of the residuals from the ephemeris registration expressed in units of pixels instead of radians) are given in Eqs. (10) and (11). These are the variances based on the scatter; internal errors can be calculated from the weights alone, by Eq. (4.31) of Bevington (1992). Note the denominators differ because there is one less degree of freedom for the occulting body than the star because a line was fit through the data, whereas for the star only a mean was determined.

$$
\begin{aligned}
& \sigma^{2}\left(\left\langle r_{r * k}\right\rangle\right)=\frac{\sum_{k=1}^{N_{f}} w_{k}\left(r_{r * k}-\left\langle r_{r * k}\right\rangle\right)^{2}}{\left(N_{f}-1\right) \sum_{k=1}^{N_{f}} w_{k}}, \\
& \sigma^{2}\left(\left\langle r_{r p k}\right\rangle\right)=\frac{\sum_{k=1}^{N_{f}} w_{k}\left[r_{r p k}-r_{r e}\left(t_{k}\right)\right]^{2}}{\left(N_{f}-2\right) \sum_{k=1}^{N_{f}} w_{k}} .
\end{aligned}
$$

It is possible that all the frames do not have usable positions for both the occulting star and occulted body (for example, if the occulting body passes too close to a field star in one night's data). If this is the case, then the number of frames, $N_{f}$, for the star and occulting body will not be the same, and care is required to implement Eqs. (10) through (12) with the proper sums.

The error in the offset term, $b_{1}$ is

$$
\sigma^{2}\left(b_{1}\right)=b_{2}^{2} \sigma^{2}\left(\left\langle r_{r p k}\right\rangle\right)\left\{\frac{N_{f} \Sigma_{k=1}^{N_{f}} w_{r k}\left(r_{r p k}-\left\langle r_{r * k}\right\rangle\right)^{2}}{\sum_{k=1}^{N_{f}} w_{r k} \Sigma_{k=1}^{N_{f}} w_{r k}\left(r_{r p k}-\left\langle r_{r * k}\right\rangle\right)^{2}-\left[\sum_{k=1}^{N_{f}} w_{r k}\left(r_{r p k}-\left\langle r_{r k}\right\rangle\right)\right]^{2}}\right\} .
$$

If we denote the factor in curly braces by $\gamma_{r}^{2}$, then we can write (for our ideal case) equations for the variance in both $\xi_{*}$ and $\eta_{*}$ :

$$
\begin{aligned}
& \sigma^{2}\left(\xi_{*}\right)=b_{2}^{2}\left[\gamma_{r}^{2} \sigma^{2}\left(\left\langle r_{r p k}\right\rangle\right)+\sigma^{2}\left(\left\langle r_{r * k}\right\rangle\right)\right], \\
& \sigma^{2}\left(\eta_{*}\right)=b_{b}^{2}\left[\gamma_{c}^{2} \sigma^{2}\left(\left\langle c_{r p k}\right\rangle\right)+\sigma^{2}\left(\left\langle c_{r * k}\right\rangle\right)\right] .
\end{aligned}
$$

Equation (13) shows how the errors in the measured occulting body and occulted star positions translates into errors in $\xi_{*}$ and $\eta_{*}$. Both terms in each equation partake of a common scale factor $\left(b_{2}\right.$ or $\left.b_{6}\right)$, and the variance in the sample means add quadratically. However, we see that the $\gamma$ factors enhance the variance due to the error in the measured body positions, increasing as the distance between the mean of the body positions is more distant from the star.

The error in the impact parameter is the error of the star position projected onto a line connecting the star at the time of closest approach to the center of the occulting body. If $\theta$ is the position angle of the star relative to the center of the body (measured from North through East) at the time of closest approach, then the error in the impact parameter (in kilometers) is given by Eq. (14), where $d$ is the distance (in kilometers) from the observer to the occulting body.

$$
\sigma(p)=d\left[\sigma^{2}\left(\xi_{*}\right) \sin ^{2} \theta+\sigma^{2}\left(\eta_{*}\right) \cos ^{2} \theta\right]^{1 / 2}
$$

Similarly, the timing error is the error component perpendicular to this, divided by the shadow velocity, $v$, at the midtime of the occultation:

$$
\sigma\left(t_{0}\right)=\frac{d}{v}\left[\sigma^{2}\left(\xi_{*}\right) \cos ^{2} \theta+\sigma^{2}\left(\eta_{*}\right) \sin ^{2} \theta\right]^{1 / 2}
$$

\section{APPLICATION TO THE TR60 OCCULTATION}

For the Tr60 occultation by Triton, $20 \mathrm{CCD}$ frames of the star field were taken on the nights of 1993 July 8-11 with the 61-in. astrometric reflector at the USNO Flagstaff Station. The detector is a Tektronix, thinned, back-side illuminated, $2048 \times 2048 \mathrm{CCD}$ with an image scale of about 0.325 arcsec per pixel (field of view equal to 11 arcmin). These frames were exposed through a broad-band red filter (USNO designation A2-1, with a passband of 6600-8300 $\AA$ ). Typical exposure times for the frames were $60 \mathrm{~s}$ (longer exposures could not be taken because Neptune would saturate).

Images with known systematic errors were not included in the analysis. The data selection process removed centers of either field stars, Tr60, or Triton from the analysis; some- 
TABLE I

Tr60 Astrometric Data, rms Error and Intensity Ratio

\begin{tabular}{|c|c|c|c|c|c|c|c|c|}
\hline \multirow[t]{2}{*}{ Date } & \multirow{2}{*}{$\begin{array}{c}\text { \# Frames } \\
\text { (Tr60/Triton) }\end{array}$} & \multirow{2}{*}{$\begin{array}{l}\text { Min. HA } \\
\text { (hrs.) }\end{array}$} & \multirow{2}{*}{$\begin{array}{l}\text { Max. HA } \\
\text { (hrs.) }\end{array}$} & \multicolumn{2}{|c|}{ Tr60 } & \multicolumn{2}{|c|}{ Triton } & \multirow{2}{*}{$\begin{array}{c}\text { Intensity Ratio } \\
\text { (Tr60/Triton) }\end{array}$} \\
\hline & & & & $\begin{array}{c}\text { Row Error } \\
\sigma\left(\left\langle r_{r^{*} k}\right\rangle\right)\end{array}$ & $\begin{array}{l}\text { Col Error }{ }^{a} \\
\sigma\left(\left\langle c_{r^{*} k}\right\rangle\right)\end{array}$ & $\begin{array}{l}\text { Row Error } \\
\left.\sigma\left(\left\langle r_{\text {rpk }}\right\rangle\right)\right)\end{array}$ & $\begin{array}{l}\text { Col Error }{ }^{\mathrm{a}} \\
\sigma\left(\left\langle c_{\text {rpk }}\right\rangle\right)\end{array}$ & \\
\hline 199 & & & 0 & 0.022 & 0.059 & 0.016 & 0.040 & 0.0006 \\
\hline 19 & 20 & .66 & 0 & 0.027 & 0.051 & 0.030 & 0.059 & 0.0005 \\
\hline 1993 July 10 & $18 / 2$ & -0.81 & 0.53 & 0.065 & 0.076 & 0.069 & 0.091 & $0.5112 \pm 0.0030$ \\
\hline 1993 July 11 & $16 / 16$ & -0.94 & 0.14 & 0.074 & 0.056 & 0.062 & 0.075 & $0.4824 \pm 0.0009$ \\
\hline
\end{tabular}

a Errors are for an individual frame based on the scatter. Internal error agrees well with error from scatter. Units are pixels and the mean focal plane scale is about 0.325 arcsec/pixel.

times entire frames were removed. Triton centers were removed either because of high residuals in the ephemeris registration (this was the case for two centers) or because Triton was merged with a field star ( 18 of the Triton centers from July 10 were not included in the analysis for this reason). More detailed modeling of the two sources may provide accurate astrometry and photometry, but for now these data are not included. Two field stars were not included in the analysis because they were too close to the edge of the CCD (less than 20 pixels $\approx 7$ arcsec) and showed high residuals in the registration. One frame was removed on the basis of photometry, since the magnitude difference between $\operatorname{Tr} 60$ and Triton was not consistent with the other frames from that night. Frames with thin cirrus as evidenced by inconsistent photometry, or field stars not identified on all frames were also left out of the reduction. To reduce the effects of refraction and telescope flexure, the observations occurred only within $1 \mathrm{~h}$ of the meridian, see Table 1 . There were 44 field stars that comprise the detector network (see Fig. 1), common to all the 74 useful frames (Table 1).

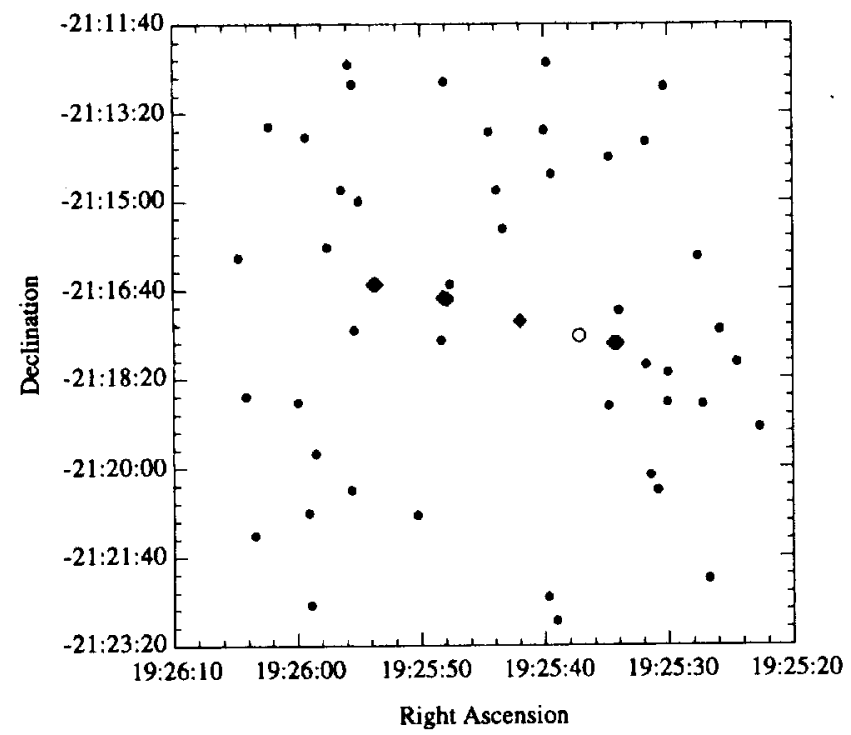

FIG. 1-Tr60 Field. The star field of the USNO stare frames is shown schematically. The 44 field stars used to construct the mean-frame coordinate system are indicated by filled circles. The occulted star, Tr60, is shown by the open circle. The motion of Triton across the field is indicated by the diamonds for the first and last observation from each night. The USNO frames were all centered to within 3 arcsec.

\subsection{Astrometry}

Before we can apply our astrometric method to the data, we must calculate centers for the field stars, Triton and Tr60. This is done by putting the flattened frames through a series of IRAF scripts that use the functions in the DAOPHOT package (Stetson 1987) to perform numerical point-spreadfunction (PSF) fitting of the observed stars. First, the routine "daofind" is used to identify all stellar images on the frame that are within a given range of brightness (neither too faint to be in the noise, nor too bright to be in the nonlinear regime of the detector). This program convolves the data with a Gaussian function, and then examines the convolved data for local maxima. Approximate centroids, as well as the roundness and sharpness of the image are calculated. These last two parameters are used to eliminate bright pixels, cosmic rays, and extended objects from the star list.

Next, aperture photometry of the stars identified by "daofind" is performed with "apphot." An IRAF script selects stars for the PSF which (i) are neither too bright nor too faint, (ii) are not near the edge of the frame, and (iii) do not have nearby neighbors. This selection is done on the basis of the aperture photometry and "daofind" results. The numerical PSF is fit to all identified stars with the DAOPHOT function "nstar" that uses a least-squares procedure to simultaneously fit stars that are close together. The result is the row and column center $(r, c)$, instrumental magnitude, and error in the instrumental magnitude for each object. The resulting lists are edited to include only: (i) the occulted star (ii) the occulting body, and (iii) the detector-network stars. Since the errors in the centers were independent of magnitude for the network stars, the weighting given by Eq. (1) was used.

We used the DE211 ephemeris to define the motion of the Neptune system's center of mass and the NEP 016 model (Jacobson et al. 1991) to define the motion of Triton relative to the center of mass of the system. Triton's ephemeris was topocentric (i.e., it included effects of geocentric parallax and light travel time), but no adjustment was added for the apparent displacement due to diurnal aberration, annual aberration, and refraction.

One could add the effects of aberration and refraction to the ephemeris, but Table 2 shows that the unmodeled contribution of these effects is negligible. Values of the maximum image displacements are given in Table 2. For each effect we have selected the frame that would have the maximum image displacement and have calculated (i) mean displacements of the images for the frame, and (ii) the maximum part of the 
TABLE 2

Maximum Image Displacements in the USNO Frames

\begin{tabular}{lcc}
\hline \multicolumn{1}{c}{ Effect } & $\begin{array}{c}\text { Mean for Frame } \\
(\operatorname{arcsec})\end{array}$ & $\begin{array}{c}\text { Not Modeled } \\
(\operatorname{arcsec})\end{array}$ \\
\hline Diurnal Aberration & 0.26 & $3.410^{-7}$ \\
Annual Aberration & 21. & $2.710^{-5}$ \\
Refraction (monochromatic) & 75. & $6.610^{-4}$ \\
Refraction (chromatic) & 0.015 & $8.610^{-4}$ \\
\hline \hline
\end{tabular}

effect that would not be modeled by the linear registration of Eq. (2). For the effects of diurnal aberration, annual aberration, and monochromatic refraction, the appropriate angle over which to propagate the unmodeled terms is half of the detector field of view. The mean image displacements due to chromatic terms in atmospheric refraction do not affect us, but the differential displacements due to this effect cause unmodeled trends in the positions of network stars that depends on their color. We have measured the colors for 13 of the 44 stars and have calculated the image displacements for a hypothetical star with the mean color. The maximum displacement (determined for the star with the most extreme difference in color and for the frame with the greatest hour angle) has been entered in Table 2. Although, these image displacements would not be removed by the linear registration of Eq. (2), most are much smaller than the maximum given in the table, and they should be of random magnitude over the network. The signature of these errors would be a systematic trend of the registration residuals as a function of hour angle, but this trend was not apparent. From the entries in Table 2 we conclude that the linear approximation for these effects would not introduce significant errors into our analysis.

However, if one still wanted to add the effects of aberration and refraction to the ephemeris, care is needed to do this properly. First one needs to calculate the amount of the displacement of the Triton center on each frame, then the displacement added to the ephemeris is the weighted average displacements of each individual frame. This is needed to be consistent with the Triton centers in the detector network coordinate system, which have a net displacement of each star by the weighted average of the star's displacement on each frame.

The residuals from registering the Triton centers to the ephemeris are shown in Fig. 2. The figure shows the residual of each Triton center as a function of its location in the detector network. The residuals have been multiplied by a factor of 100 . Note that no systematic trends are evident. The weighted rms residual is 0.001 arcsec in the $\xi$ direction and 0.003 arcsec in the $\eta$ direction.

The standard error of the Tr60 center and the equivalent for the Triton centers (as expressed in the error discussion) are listed for each night's observations in Table 1. As seen in the table, there is a nightly variation of the data quality. These numbers also show that the scatter in the Triton position is not inflated due to the presence of Neptune.

Using the method outlined in Sec. 3, we derived a minimum distance for the KAO from the center of the shadow of $359 \pm 133 \mathrm{~km}(0.017 \pm 0.006 \mathrm{arcsec})$; the center of Triton's shadow was north of the KAO, see Fig. 3. As an independent check of the astrometric solution's accuracy, we can compare the observed midtime of the occultation with the closest approach time from astrometry. For this event, the astrometric closest approach time was $2.2 \mathrm{~s}$ before the midtime from the light curve. This time difference is less than the formal error in the timing $(4.1 \mathrm{~s}$ ) and translates to 60 kilometers (using Triton's shadow velocity of $27.37 \mathrm{~km} \mathrm{~s}^{-1}$ for the KAO), which is less than the formal error on the miss distance. The latitudes probed at the half-light level of immersion and emersion are also listed in Table 3. The full set of latitudes probed by this occultation chord are displayed in the right panel of Fig. 4. Note that the occultation probes Triton's southern latitudes [those latitudes seen by Voyager (Smith et al. 1989)]. The left panel of Fig. 4 shows Triton's globe and the path of the star relative to Triton as seen by the KAO.

Figure 5 is a plot in the tangent plane of the residuals from the registration of the field stars to a secondary astrometric network supplied by A. Klemola (1993, personal communication). This network was a re-reduction (using the

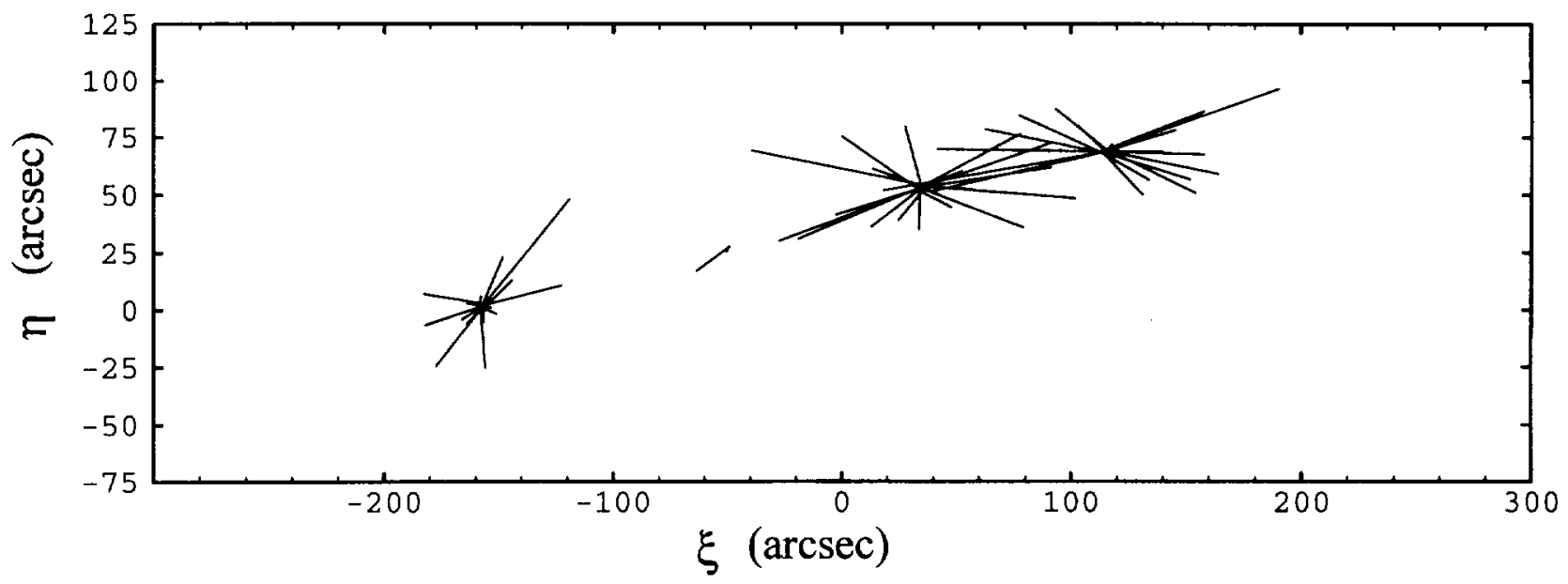

FIG. 2-Triton registration residuals. The residuals (muitiplied by a factor of 100) from the registration of the Triton centers to the ephemeris are displayed. Each residual is indicated by a line whose origin is the Triton position for that frame. 


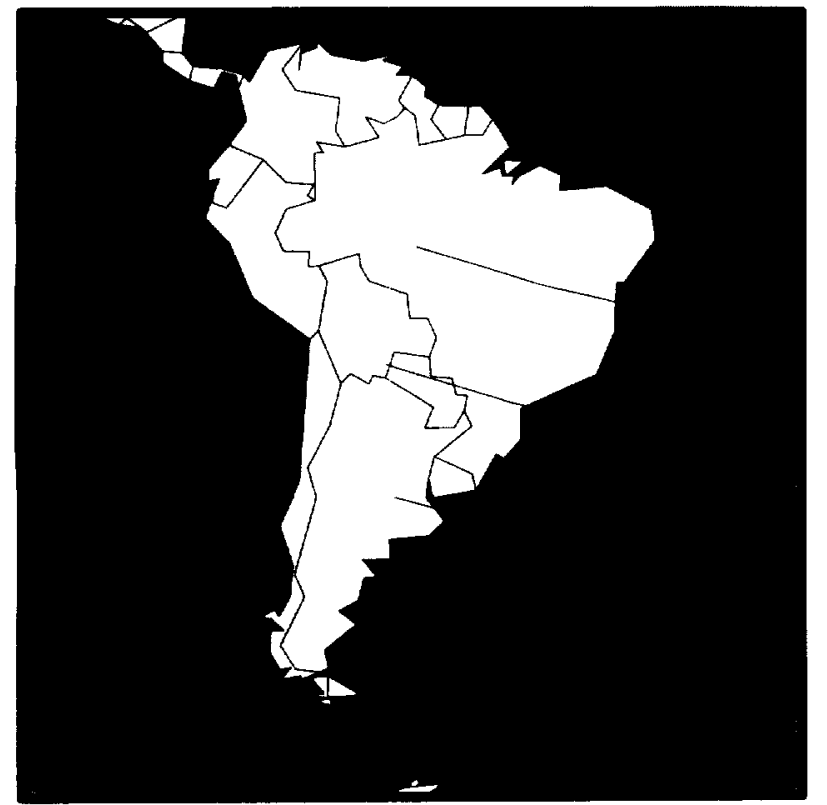

FIG. 3-Triton's Shadow Path and the KAO. The northern limit, centerline, and southern limit of Triton's shadow are shown as they crossed South America. The flight of the Kuiper Airbome Observatory, indicated by the bold line, starts at Punta Arenas, Chile and ends in Buenos Aires, Argentina. Occulation series images were obtained for 35 min around the occultation time on the south-bound leg of the flight.

ACRS network) of plates taken to search for Neptune candidates (Klemola and Mink 1991) originally reduced with the Perth 70 catalog. The residuals have been multiplied by a factor of 100 so that they may be visible in the figure. Note that there are no obvious systematic trends in the residuals. Since we are only concerned with relative positions, systematic errors in the absolute position (which may not be apparent in this figure) are not relevant. The standard deviation of these residuals are 0.18 aresec in the $\xi$ direction and 0.13 arcsec in the $\eta$ direction.

\subsection{Photometry}

The accurate analysis of an occultation light curve relies not only on an astrometric calibration but a photometric one too. The photometric calibration involves determining the relative magnitudes of the occulted star and occulting body to define the zero-flux level of the light curve. For the Tr60 occulation, the relative magnitudes of the objects were derived from the same USNO data on which the astrometric solution is based. There were other sources of photometric

TABLF: 3

Tr60 Occultation Circumstances

\begin{tabular}{lc}
\hline \hline Impact Parameter for the $\mathrm{KAO}(\mathrm{km})$ & $359 \pm 133$ \\
Time Difference $(\mathrm{s})$ & $2.2 \pm 4.1$ \\
Impact parameter f component $(\mathrm{km})$ & $77 \pm 111$ \\
Impact parameter $\mathrm{g}$ component $(\mathrm{km})$ & $-350 \pm 134$ \\
shadow velocity for the KAO $(\mathrm{km} / \mathrm{s})$ & 27.37 \\
Immersion half-light latitude $(\mathrm{deg})$ & $-28.9(+2.6,-2.1)$ \\
Emersion half-light latitude (deg.) & $3.6(+4.5,-5.2)$ \\
\hline \hline aight curve occultation midtime minus astrometric closcst \\
approach time
\end{tabular}

data, but due to nonlinearities in other detectors, these data are the best source for the photometric calibration.

The flux ratios of $\operatorname{Tr} 60$ to Triton on a nightly basis are listed in Table 1 and are displayed in Fig. 6. There is variability in the relative intensities, either from variability in Triton as a function of rotational phase or from the variability of $\operatorname{Tr} 60$. To get the intensity ratio at the occultation time, we linearly interpolated using the last three nights of data (as defined by the midtime of the light curve). This photometric determination of the relative intensities involves a different instrument and filter than the occultation observations. The effective wavelength of each system (KAO and USNO) was determined from the quantum efficiency of each detector and the filter (no filter was used for the KAO observations).

The effect of refraction on the effective wavelength was ignored. The constant of refraction changes by less than $1 \%$ over the wavelength of the filter used at the USNO. At the altitude of the KAO, the atmospheric pressure is lower than sea level by about a factor of 5 , and the change in refraction over the wavelengths of the instrument (the NASA/Ames Portable CCD) sensitivity is only $1 \% . R$ and $I$ photometry of Tr60 ( $R=13.39 \pm 0.02 ; I=13.82 \pm 0.02 ;$ D. M. Chamberlain, personal communication) and Triton (Buratti et al. 1994) provided the slope of the intensity ratio with wavelength from the effective wavelength of the USNO observations $(0.65 \mu \mathrm{m})$ to the effective wavelength of the KAO observations $(0.67 \mu \mathrm{m})$, see Table 4 . The $R$ and $I$ magnitudes for Tr60 are approximately Kron-Cousins (M. Buie, personal communication).

\section{DISCUSSION}

The method outlined in this paper to determine the closest approach distance between a star and a body passing close by has been applied to the 1993 Triton occultation, and we find consistency between the closest approach time predicted by the astrometry and that derived from the occultation light curve. To the level of accuracy presented, we find no systematic problems with either the analysis or the data. Much of this is due to the high astrometric quality of the telescope and detector, as well as the practice of observing only when the objects are near transit.

Now we consider the sources of formal error. Note that choosing the occulted star for the origin of the detectornetwork coordinate system simplifies the error analysis, so that only the errors in the offset terms of the registration and the scatter of the star center [the two parts of Eq. (13)] contribute. For this dataset, we are limited by the error in the registration coefficients $\left(\sigma\left(b_{1}\right)=0.005\right.$ arcsec and $\sigma\left(b_{4}\right)=0.006$ arcsec), not the scatter in the Tr60 center $(0.001$ arcsec in $\xi$ and $0.002 \operatorname{arcsec}$ in $\eta$ ). Therefore, more observations of the star would not significantly improve the solution. Improving the precision of the solution could have been accomplished by (i) more observations of Triton (to reduce the registration coefficient error), which cannot be increased arbitrarily because the occulting body only spends a finite amount of time in the same field with the occulted star, and (ii) arranging the observations so that the mean Triton position were close to the $\operatorname{Tr} 60$ position. If this were true for the present data set, then the $\gamma$ factor in Eq. (13) 

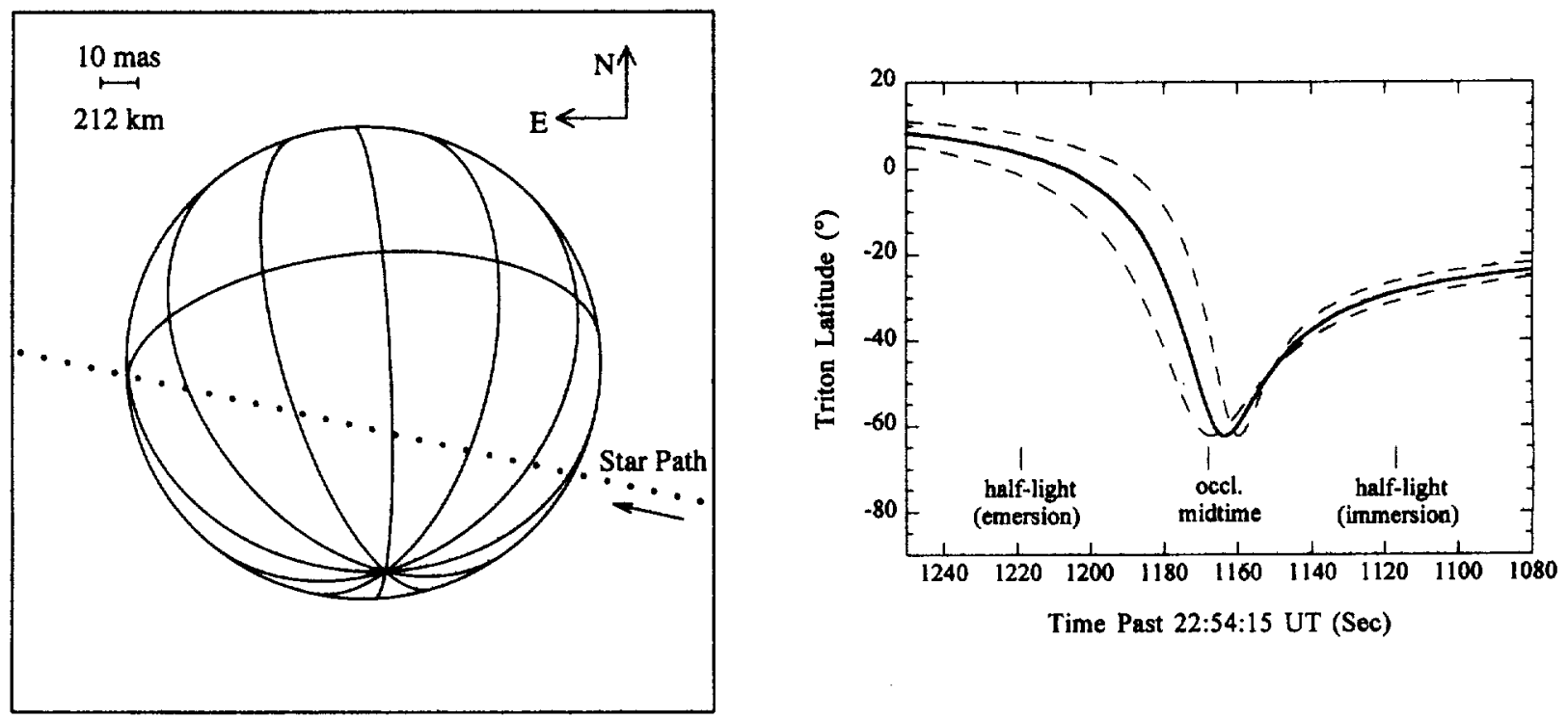

FIG. 4-The region of Triton probed by this stellar occultation. The left panel shows Triton as seen by the KAO. Triton's south pole is visible. The path of the star relative to Triton is indicated at 5-s intervals. The right panel shows the latitude of Triton that was probed as a function of time. The dashed lines indicate the \pm 1 -sigma limits on the latitudes probed. Note that the time axis is reversed to be consistent with the path of the star in the left panel. The midtime of the occultation, along with the half-light immersion and emersion times from the light curve are indicated. The occultation probed the southern hemisphere during the midtime of the occultation.

would have been unity and the error in the minimum separation would have been reduced from 0.006 to 0.003 arcsec. For this analysis, the effective mean Triton position was further from the $\operatorname{Tr} 60$ position than a simple (unweighted) average would indicate, because the first two night's data (both recorded before the event) were the highest quality.

Outer solar system bodies such as Triton and Pluto are on the same 11 arcmin field as a given candidate star for about

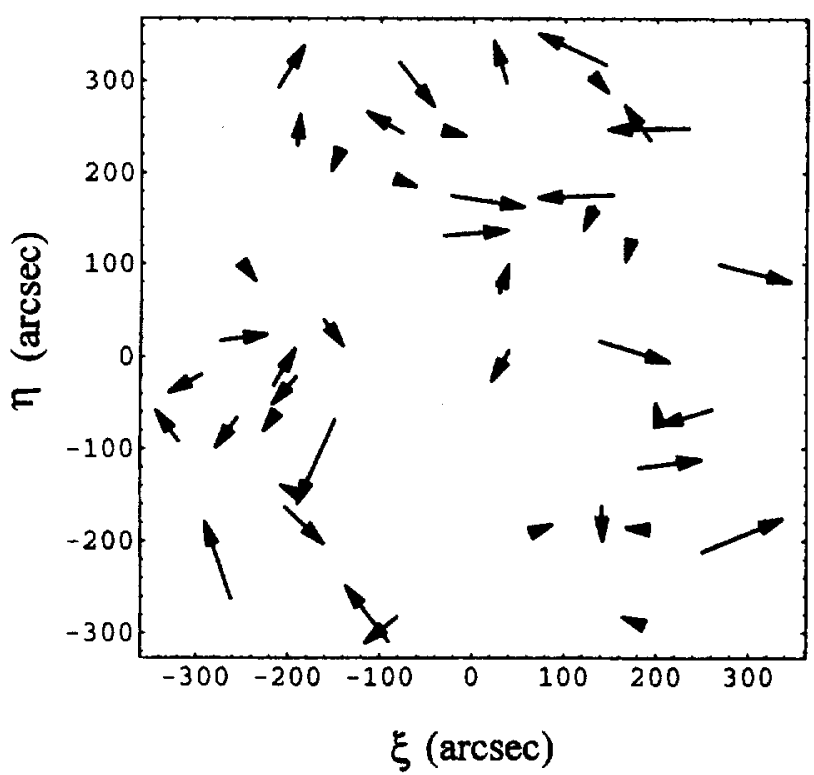

FIG. 5-The residuals from registering the weighted mean frame to a secondary astrometric network. The residuals have been multiplied by a factor of 100 . Each of the standard stars are indicated in the figure by their $(\xi, \eta)$ components, and their residual components are indicated by the arrow. one week at opposition. However, less remote bodies generally will move through the field more quickly, decreasing the window of opportunity for obtaining this critical calibration data. For the most accurate results, the observations should be taken both before and after the occultation, so that we can interpolate to derive the closest approach distance (rather than extrapolate) and to minimize the $\gamma$ factor in Eq. (13).

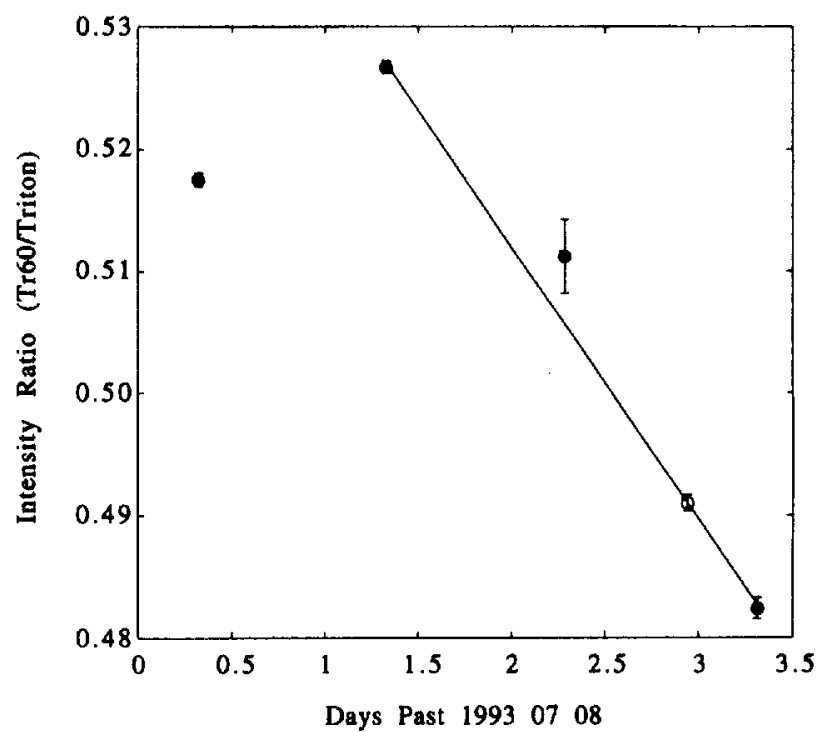

FIG. 6-The relative intensity of $\operatorname{Tr} 60$ and Triton as a function of time. The filled circles indicate the intensity ratio for each night's observations with an error bar given by the standard error of the sample mean. There were only two good Triton observations on the third night, hence the error bar is larger. Also shown in the figure is the best fit line to the observations from the last three nights. The open circle represents the adopted intensity ratio measured with the USNO system for the time of the occultation. 
TABLE 4

Tr60 Photometry

\begin{tabular}{ccc}
\hline \hline Dataset & $\begin{array}{c}\text { effective } \lambda \\
(\mu \mathrm{m})\end{array}$ & $\begin{array}{c}\text { Intensity Ratio } \\
\text { (Tr60/Triton) }\end{array}$ \\
\hline USNO & 0.67 & $0.4910 \pm 0.0007$ \\
KAO & 0.65 & $0.4895 \pm 0.0012^{\text {a }}$ \\
\hline \hline
\end{tabular}

${ }^{a}$ derived from the USNO intensity ratio and the effective wavelengths

This method provides the following two advantages in comparison to the standard astrometric reduction technique which is commonly used for occultation predictions (Wasserman et al. 1979). First, no astrometric network is needed. With CCD data, the field is generally small enough that primary astrometric networks do not have a sufficient number of standard stars. So eliminating the need for the dense astrometric network saves significant time and effort. The second advantage is that this method is immune to scale and rotation errors in an astrometric network that result from the random errors in the star positions and any systematic differences between the stellar reference frame and that of the ephemerides. We do not expect to find significant scale errors in the planetary motion (which sets the scale) as defined by ephemerides, because this error would accumulate over time and an offset of the body from its ephemeris would be noticed.

This method has the disadvantage that it cannot be used for all occultations. If the occulting body has a linear path across the detector, then the motion of the body can only set the scale in the direction of motion, and an astrometric network is needed to define the perpendicular scale. This "compromise" method was used in the 1994 March 9 occultation by Chiron (Elliot et al. 1995).

Over time, this ephemeris method can be employed to determine a "catalog" of star positions in the reference frame of planetary ephemerides. This catalog could then be used to investigate differences between the reference system of ephemerides and stellar reference frames. Toward this end, we present three positions for $\operatorname{Tr} 60$ (all in J2000) based on three different astrometric reductions, see Table 5. These positions come from the ephemeris method and from astrometric reductions of plates (Klemola 1993, personal communication) using two separate primary astrometric networks: the ACRS and Perth 70. The coordinates of the detectornetwork stars are not given here because the right ascension

TABLE 5

Tr60 Positions (J2000 FK5)

\begin{tabular}{lcc}
\hline Reference System & RA & Dec \\
\hline Triton ephemeris & 192537.227 & -211732.66 \\
ACRS network & 192537.256 & -211732.70 \\
Perth 70 network & 192537.205 & -211732.73 \\
\hline
\end{tabular}

and declination of these stars were not determined as accurately as Tr60 since Triton did not pass close to these other stars. Many of the stars would be outside of Triton's path, and the image scale would have to be extrapolated to their locations, which introduces errors.

For the most precise determination of an occultation chord location, it is best to have well spaced, multiple chords. However, with occultations by small bodies it is not always possible to have multiple observers in the shadow path (due to the uncertainties of the prediction, even a week in advance). Useful information can be derived from these single-chord occultation observations with the ephemeris method presented here, if there are quality astrometric data of the occulted star and occulting body both before and after the occultation.

We thank Dave Monet and James Bauer for taking two nights of data at the USNO. We are grateful to Amanda Bosh and Dawn Chamberlain for supplying photometry of Tr60 and would like to thank Arnold Klemola for providing two secondary astrometric networks for this analysis. Fred Harris kindly supplied the QE curve of the USNO CCD. This work was supported, in part, by NASA Grants Nos. NAG2-836, NAG2-903, NAG2-811, NAGW-1494.

\section{REFERENCES}

Baron, R. L., French, R. G., and Elliot, J. L. 1989, Icarus, 78, 119 Bevington, P. R., and Robinson, D. K. 1992, Data Reduction and Error Analysis for the Physical Sciences (New York, McGrawHill)

Buratti, B. J., Goguen, J. D., Gibson, J., and Mosher, J. 1994, Icarus 110,303

Clifford, A. A. 1973, Multivariate Error Analysis (London, Applied Science Publishers, Ltd.)

Dunham, D. W., et al. 1990, AJ, 99, 1636

Dunham, E. W., McDonald, S. W., and Elliot, J. L. 1991, AJ, 102, 1464

Elliot, J. L. 1979, ARAA, 17, 445

Elliot, J. L., et al. 1993, AJ, 106, 2544

Elliot, J. L., et al. 1995, Nature, 373, 46

French, R. G., et al. 1988, Icarus, 73, 349

French, R. G., et al, 1985, AJ, 90, 2624

Jacobson, R. A., Riedel, J. E., and Taylor, A. H. 1991, A\&A, 247, 565

Klemola, A. R., \& Mink, D. J. 1991, AJ, 102, 389

Lellouch, E., Hubbard, W. B., Sicardy, B., Vilas, F., and Bouchet, P. 1986, Nature 324, 227

McDonald, S. W., and Elliot, J. L. 1992, AJ, 104, 862

Millis, R. L., et al. 1993, Icanus, 105, 282

Nicholson, P. D., McGhee, C., and French, R. G. 1995, Icarus, 113, 57

Sicardy, B., Roques, F., and Brahic, A. 1991, Icarus, 89, 220

Smart, W. M. 1977, Textbook on Spherical Astronomy (Cambridge, Cambridge University Press)

Smith, B. A., et al. 1989, Science, 246, 1422

Stetson, P. B. 1987, PASP, 99, 191

Wasserman, L. H., et al. 1979, AJ, 84, 259 
